QUARTERLY OF APPLIED MATHEMATICS

VOLUME LXVIII, NUMBER 1

MARCH 2010, PAGES 17-42

S 0033-569X(09)01165-9

Article electronically published on October 15, 2009

\title{
GRAVITATIONAL COLLAPSE AND THE FORMATION OF BLACK HOLES FOR THE SPHERICALLY SYMMETRIC EINSTEIN-VLASOV SYSTEM
}

\author{
BY \\ HÅKAN ANDRÉASSON (Mathematical Sciences, Chalmers University of Technology, Göteborg \\ University, S-41296 Göteborg, Sweden), \\ MARKUS KUNZE (Fachbereich Mathematik, Universität Duisburg-Essen, D-45117 Essen, \\ Germany), \\ AND \\ GERHARD REIN (Mathematisches Institut der Universität Bayreuth, D-95440 Bayreuth, Germany) \\ Dedicated to Prof. W. A. Strauss on the occasion of his 70th birthday
}

\begin{abstract}
We review results on the spherically symmetric, asymptotically flat Einstein-Vlasov system. We focus on a recent result where we found explicit conditions on the initial data which guarantee the formation of a black hole in the evolution. Among these data there are data such that the corresponding solutions exist globally in Schwarzschild coordinates. We put these results into a more general context, and we include arguments which show that the spacetimes we obtain satisfy the weak cosmic censorship conjecture and contain a black hole in the sense of suitable mathematical definitions of these concepts which are available in the literature.
\end{abstract}

\section{Contents}

1. Some general remarks on gravitational collapse

2. The Einstein-Vlasov system

3. Local and global existence results

3.1. Local existence and continuation

3.2. Global existence for small data

3.3. Possible breakdown must occur at the center first

4. Gravitational collapse and formation of black holes

Received December 9, 2008.

2000 Mathematics Subject Classification. Primary 35Q75; Secondary 83C75, 85A05.

Key words and phrases. General relativity, Einstein-Vlasov system, gravitational collapse, black holes. Support of the first author by the Institut Mittag-Leffler (Djursholm, Sweden) is gratefully acknowledged.

E-mail address: hand@math.chalmers.se

E-mail address: markus.kunze@uni-due.de

E-mail address: gerhard.rein@uni-bayreuth.de 
4.1. The system on an outer domain

4.2. The main result - analysis in Schwarzschild coordinates

4.3. Weak cosmic censorship and the formation of a black hole

5. Concluding remarks

5.1. General matter models

5.2. Related results

5.3. Open problems and future perspectives

Acknowledgement

References

1. Some general remarks on gravitational collapse. We begin these notes with some general, informal, and in part historical remarks on concepts related to the phenomenon of relativistic gravitational collapse.

Shortly after A. Einstein published his theory of general relativity [24, 25], K. Schwarzschild showed [49] that the following metric solves the corresponding field equations in vacuum:

$$
d s^{2}=-\left(1-\frac{2 M}{r}\right) d t^{2}+\left(1-\frac{2 M}{r}\right)^{-1} d r^{2}+r^{2}\left(d \theta^{2}+\sin ^{2} \theta d \varphi^{2}\right) .
$$

Here $M>0$ is a parameter, $t \in \mathbb{R}$ is a time coordinate, the spacetime is spherically symmetric, and the polar angles $\theta$ and $\varphi$ coordinatize the surfaces of constant $t$ and $r>0$. The latter are the orbits of $\mathrm{SO}(3)$ which acts isometrically on this spacetime, and $4 \pi r^{2}$ is the area of these surfaces. The part of this metric with $r>2 M$ can be thought of as representing the gravitational field outside a static, spherically symmetric body of mass $M$ and radius larger than $2 M$. With this interpretation in mind, the radii $r=2 M$ and $r=0$, where the metric looks singular, lie within the matter where the metric does not apply anyway so that one need not worry about this singular behavior. However, using the new time coordinate

$$
\tilde{t}:=t+r^{*}, \text { where } r^{*}:=r+2 M \ln (r-2 M),
$$

the metric takes the form

$$
d s^{2}=-\left(1-\frac{2 M}{r}\right) d \tilde{t}^{2}+2 d \tilde{t} d r+r^{2}\left(d \theta^{2}+\sin ^{2} \theta d \varphi^{2}\right),
$$

which extends smoothly through $r=2 M$; i.e., $r=2 M$ is only a coordinate singularity in (1.1). On the other hand, the so-called Kretschmann scalar

$$
K:=R^{\alpha \beta \gamma \delta} R_{\alpha \beta \gamma \delta}
$$

derived from the Riemann curvature tensor $R_{\alpha \beta \gamma}{ }^{\delta}$ blows up like $M^{2} / r^{6}$ as $r \rightarrow 0$; Greek indices are running from 0 to 3 and are summed over if they appear as both lower and upper indices in the same expression. Since $K$ is a scalar quantity, its values do not change under a change of coordinates. The singularity at $r=0$ is a genuine feature of the Schwarzschild spacetime which cannot be cured away by a more judicious choice of coordinates; it is a spacetime singularity, where the structure of spacetime itself breaks down. Although the surface $r=2 M$ is not singular, there is something special about 
it, as can be seen from (1.3). The line $r=2 M, \theta=\pi / 2, \varphi=0$ represents the world line of a massless particle (a photon) moving radially outward, away from the origin. Since no material particle can move faster than light, particles and photons can only pass inward through the surface $r=2 M$, but can never leave the region $r<2 M$ once they are inside. Such a surface was later termed an event horizon. One should keep in mind from the above that by switching to different coordinates one was able to extend the spacetime beyond the region covered by Schwarzschild coordinates, even though in the latter coordinates the metric blows up at the boundary $r=2 M$.

While the part with $r>2 M$ of the Schwarzschild metric was successfully used to explain for example the perihelion precession of Mercury in the solar system, it was argued that no conceivable physical process could compress an amount of matter so much that its mass $M$ would all be contained inside the region $r<2 M$, and so the irritating behavior at the surface $r=2 M$ and also the breakdown of the geometry of spacetime at $r=0$ was discarded as unphysical.

But in 1939, J. R. Oppenheimer and H. Snyder [32] constructed a semi-explicit, timedependent solution of the Einstein field equations where a homogeneous spherically symmetric ball of dust, i.e., of a fluid with pressure zero, collapses until all the mass is within the region $r<2 M$, and it continues to collapse until the scalar curvature of spacetime blows up at the center of symmetry. Although this proved that an event horizon can evolve out of completely regular initial data, several decades passed before such structures became accepted as potentially relevant from the physics point of view and J. A. Wheeler coined the name black hole for them.

Today, there are many astronomical observations for which the currently best explanation involves a black hole. For example, black holes of the order of $10^{6}-10^{9}$ solar masses are believed to reside in the centers of many galaxies, including the Milky Way. In spite of the increasing relevance of black holes as real astrophysical objects, many important basic questions about gravitational collapse are still open. The most prominent of these is the cosmic censorship conjecture.

To see the issue here, we notice that in the Oppenheimer-Snyder example, the spacetime singularity which forms at $r=0$ is hidden behind the event horizon so that it cannot be seen or in any other way be experienced by observers outside the event horizon. The same is true for the spacetime singularity in the Schwarzschild spacetime. In the 1960s, R. Penrose 33 . proved that a spacetime singularity forms in the gravitational collapse of a not necessarily symmetric star made up of "reasonable" matter, i.e., matter which satisfies the strong energy condition, provided a closed trapped surface forms. This is a closed, two-dimensional, spacelike surface with the property that the null geodesics, i.e., the light rays, which start perpendicular to the surface decrease its surface area both when followed inward and outward from the surface; for a precise definition, cf. [51, 9.5]. The surfaces of constant $\tilde{t}$ and $r$ with $r<2 M$ in (1.3) are trapped, and we note that Schwarzschild coordinates cannot cover regions of spacetime which contain trapped surfaces. Since trapped surfaces are stable under small perturbations of the spacetime, Penrose's result showed that the formation of spacetime singularities is not restricted to spherically symmetric, especially constructed or isolated examples but is a genuine, 
stable feature of spacetimes. However, his result gives little information about the geometric structure of a spacetime with such a singularity; in particular, it does not provide an event horizon surrounding the singularity. The existence of a naked singularity, which by definition is not hidden behind an event horizon, would violate predictability as it would not be possible to predict from initial data what an observer would see if he could observe a singularity. Hence Penrose formulated the cosmic censorship conjecture which demands that any singularity arising in the gravitational collapse of generic regular initial data is hidden behind an event horizon; exceptional data leading to naked singularities are required to form a "null set" in some suitable sense. The above is an informal statement of the so-called weak cosmic censorship conjecture [51, 12.1]. It would in particular guarantee that predictability holds at least in the region outside the event horizon. In the strong version, no observer is allowed to observe a singularity. For a mathematical definition and discussion of the weak and strong cosmic censorship conjectures, we refer to $[20$.

An important example where naked singularities do form for a null set of data while cosmic censorship holds for generic data is the spherically symmetric Einstein-scalar field system which was investigated by D. Christodoulou; cf. [18, 19. A massless scalar field or dust as employed by Oppenheimer and Snyder and later also by Christodoulou [13] are but two possibilities for modeling matter in gravitational collapse. In the present notes we discuss results where a collisionless gas as described by the Vlasov equation is used as matter model, a model which we consider particularly suitable for this purpose from a mathematics point of view and which is well motivated from an astrophysics point of view; cf. [11.

2. The Einstein-Vlasov system. Consider a smooth spacetime manifold $M$ equipped with a Lorentzian metric $g_{\alpha \beta}$ with signature $(-+++)$. The Einstein equations read

$$
G_{\alpha \beta}=8 \pi T_{\alpha \beta},
$$

where $G_{\alpha \beta}$ is the Einstein tensor, a nonlinear second-order differential expression in the metric $g_{\alpha \beta}$, and $T_{\alpha \beta}$ is the energy-momentum tensor given by the matter content (or other fields) of the spacetime. To obtain a closed system, the field equations (2.1) have to be supplemented by evolution equation(s) for the matter and the definition of $T_{\alpha \beta}$ in terms of the matter and the metric.

We choose as a matter model a collisionless gas. In order to write down an evolution equation for the number density of the particles on phase space we recall that the world line of a single test particle on $M$ obeys the geodesic equation

$$
\dot{x}^{\alpha}=p^{\alpha}, \dot{p}^{\alpha}=-\Gamma_{\beta \gamma}^{\alpha} p^{\beta} p^{\gamma},
$$

where $x^{\alpha}$ denotes general coordinates on $M, p^{\alpha}$ are the corresponding canonical momenta, $\Gamma_{\beta \gamma}^{\alpha}$ are the Christoffel symbols induced by the metric $g_{\alpha \beta}$, and the dot indicates differentiation with respect to an affine parameter, i.e., with respect to proper time along the world line of the particle. We assume that all the particles have the same rest mass, normalized to unity, and move forward in time. Hence, their number density $f$ is a 
nonnegative function supported on the mass shell

$$
P M:=\left\{g_{\alpha \beta} p^{\alpha} p^{\beta}=-1, p^{0}>0\right\},
$$

a submanifold of the tangent bundle $T M$ of the spacetime manifold $M$ which is invariant under the geodesic flow. Letting Latin indices range from 1 to 3 we use coordinates $\left(t, x^{a}\right)$ with zero shift, which implies that $g_{0 a}=0$. On the mass shell $P M$ the variable $p^{0}$ then becomes a function of the remaining variables $\left(t, x^{a}, p^{b}\right)$ :

$$
p^{0}=\sqrt{-g^{00}} \sqrt{1+g_{a b} p^{a} p^{b}} .
$$

Since the particles move like test particles in the given metric, their number density $f=f\left(t, x^{a}, p^{b}\right)$ is constant along the geodesics and it satisfies the Vlasov equation

$$
\partial_{t} f+\frac{p^{a}}{p^{0}} \partial_{x^{a}} f-\frac{1}{p^{0}} \Gamma_{\beta \gamma}^{a} p^{\beta} p^{\gamma} \partial_{p^{a}} f=0 .
$$

The energy-momentum tensor is given by

$$
T_{\alpha \beta}=\int p_{\alpha} p_{\beta} f|g|^{1 / 2} \frac{d p^{1} d p^{2} d p^{3}}{-p_{0}},
$$

where $|g|$ denotes the modulus of the determinant of the metric, and indices are raised and lowered using the metric, i.e., $p_{\alpha}=g_{\alpha \beta} p^{\beta}$. The system (2.1), (2.4), (2.5) is the Einstein-Vlasov system in general coordinates. An introduction to relativistic kinetic theory and the Einstein-Vlasov system can be found in [1, 47. The Vlasov equation is widely used as a matter model in astrophysics to describe galaxies or globular clusters [11. Such systems are usually dealt with as isolated systems in an otherwise empty universe, which in our context means that the spacetime is asymptotically flat.

Let us for a moment consider a distribution function of the form

$$
f\left(t, x^{a}, p^{b}\right)=-u_{0}|g|^{-1 / 2} \rho\left(t, x^{a}\right) \delta\left(p^{b}-u^{b}\left(t, x^{a}\right)\right),
$$

where $\rho=\rho\left(t, x^{a}\right)$ is a scalar function on spacetime, $\delta$ is the Dirac $\delta$-distribution, and $u^{\beta}=u^{\beta}\left(t, x^{a}\right)$ takes values in the mass shell so that $u_{0}$ is determined by $u^{b}$; cf. (2.3). Then the macroscopic quantities $\rho$ and $u^{a}$ together with the metric satisfy the EinsteinEuler system for a perfect fluid with pressure zero, a matter model referred to as dust in the first section and used by Oppenheimer and Snyder; $\rho$ is the mass-energy density of the dust-fluid and $u^{\beta}$ its four-velocity. It should be stressed that although formally the Einstein-dust system can be viewed as a special case of the Einstein-Vlasov system, we use the term Vlasov matter exclusively for genuine (and usually smooth) distribution functions on the mass shell $P M$.

Before we proceed a few advantageous features of the Einstein-Vlasov system are worth pointing out. Firstly, if the metric and therefore the Christoffel symbols are given, the evolution equation for the matter, i.e., the Vlasov equation, does not produce any singularities by itself; it is, for a given metric, indeed a linear first-order conservation law which can be solved by the method of characteristics. This situation is different if the matter is described as a fluid, and singularities induced by the matter model can prevent one from analyzing the formation of event horizons and true spacetime singularities. Secondly, in the Newtonian limit the Einstein-Vlasov system turns into the Vlasov-Poisson system [40, 46] for which there is a global existence result for general, 
regular data; cf. [30, 34, 38. Hence any singularity in the solutions of the EinsteinVlasov system should have its origin in some relativistic effect; for a fluid this is again different. Beside these mathematical properties the Vlasov equation also has a clear physical interpretation and motivation, as pointed out above.

The questions raised in the previous section are at present out of reach of a rigorous mathematical treatment, unless simplifying symmetry assumptions are made. Hence we will consider the Einstein-Vlasov system under the assumption of spherical symmetry. Notice that the investigations which we cited above and where dust or a massless scalar field are used as matter models employ the same symmetry assumption. We use Schwarzschild coordinates $(t, r, \theta, \varphi)$ and write the metric in the form

$$
d s^{2}=-e^{2 \mu(t, r)} d t^{2}+e^{2 \lambda(t, r)} d r^{2}+r^{2}\left(d \theta^{2}+\sin ^{2} \theta d \varphi^{2}\right)
$$

as to the range and meaning of these coordinates, we refer to the previous section; cf. (1.1). Asymptotic flatness means that the metric quantities $\lambda$ and $\mu$ have to satisfy the boundary conditions

$$
\lim _{r \rightarrow \infty} \lambda(t, r)=\lim _{r \rightarrow \infty} \mu(t, r)=0
$$

so that as $r \rightarrow \infty$ the metric approaches the flat Minkowski metric, written in polar coordinates. In addition we impose the boundary condition

$$
\lambda(t, 0)=0
$$

in order to guarantee a regular center. Since polar coordinates sometimes induce artificial coordinate singularities at $r=0$, it is convenient to introduce the corresponding Cartesian coordinates

$$
x=\left(x^{1}, x^{2}, x^{3}\right)=r(\sin \theta \cos \varphi, \sin \theta \sin \varphi, \cos \theta) .
$$

If $p=\left(p^{1}, p^{2}, p^{3}\right)$ denotes the corresponding canonical momenta, then

$$
p_{0}=-e^{\mu} \sqrt{1+|p|^{2}\left(e^{2 \lambda}-1\right)+\left(\frac{x \cdot p}{r}\right)^{2}} \text {, }
$$

where $|p|^{2}=\left(p^{1}\right)^{2}+\left(p^{2}\right)^{2}+\left(p^{3}\right)^{2}$ and $x \cdot p=x^{1} p^{1}+x^{2} p^{2}+x^{3} p^{3}$. Since this quantity appears in the formula for the components of the energy-momentum tensor, which in turn appear as source terms in the field equations, it is preferable to use the noncanonical momentum variables

$$
v^{a}=p^{a}+\left(e^{\lambda}-1\right) \frac{x \cdot p}{r} \frac{x^{a}}{r}, a=1,2,3
$$

In these variables,

$$
p_{0}=-e^{\mu} \sqrt{1+|v|^{2}},
$$

and $f$ is spherically symmetric iff

$$
f(t, x, v)=f(t, A x, A v), x, v \in \mathbb{R}^{3}, A \in \mathrm{SO}(3) .
$$


The spherically symmetric, asymptotically flat Einstein-Vlasov system takes the following form:

$$
\begin{gathered}
\partial_{t} f+e^{\mu-\lambda} \frac{v}{\sqrt{1+|v|^{2}}} \cdot \partial_{x} f-\left(\partial_{t} \lambda \frac{x \cdot v}{r}+e^{\mu-\lambda} \partial_{r} \mu \sqrt{1+|v|^{2}}\right) \frac{x}{r} \cdot \partial_{v} f=0, \\
e^{-2 \lambda}\left(2 r \partial_{r} \lambda-1\right)+1=8 \pi r^{2} \rho, \\
e^{-2 \lambda}\left(2 r \partial_{r} \mu+1\right)-1=8 \pi r^{2} p, \\
\partial_{t} \lambda=-4 \pi r e^{\lambda+\mu} j, \\
e^{-2 \lambda}\left(\partial_{r}^{2} \mu+\left(\partial_{r} \mu-\partial_{r} \lambda\right)\left(\partial_{r} \mu+\frac{1}{r}\right)\right)-e^{-2 \mu}\left(\partial_{t}^{2} \lambda+\partial_{t} \lambda\left(\partial_{t} \lambda-\partial_{t} \mu\right)\right)=4 \pi q,
\end{gathered}
$$

where

$$
\begin{aligned}
\rho(t, r) & =\rho(t, x)=\int \sqrt{1+|v|^{2}} f(t, x, v) d v, \\
p(t, r) & =p(t, x)=\int\left(\frac{x \cdot v}{r}\right)^{2} f(t, x, v) \frac{d v}{\sqrt{1+|v|^{2}}}, \\
j(t, r) & =j(t, x)=\int \frac{x \cdot v}{r} f(t, x, v) d v, \\
q(t, r) & =q(t, x)=\int\left|\frac{x \times v}{r}\right|^{2} f(t, x, v) \frac{d v}{\sqrt{1+|v|^{2}}} .
\end{aligned}
$$

For a detailed derivation of these equations we refer to [37. It should be noted that in this formulation no raising and lowering of indices using the metric appears anywhere. It is a completely explicit system of PDEs, where $x, v \in \mathbb{R}^{3}, x \cdot v$ denotes the Euclidean scalar product, and $|v|^{2}=v \cdot v$.

For the spherically symmetric Einstein-dust equations, Christodoulou [13. showed that cosmic censorship is violated. Indeed, not even a suitable smallness condition on the initial data prevents the formation of naked singularities for dust. As we shall see in the next section this is different for the Vlasov matter model, provided we have a genuine, smooth distribution function with respect to $x$ and $v$.

The goal for the spherically symmetric, asymptotically flat Einstein-Vlasov system is to show that for all (or at least for all generic) regular initial data the corresponding solution is either global in the sense that the spacetime is singularity-free or the solution undergoes a gravitational collapse in which a spacetime singularity forms which is hidden behind an event horizon. Of course the ultimate goal would be to prove this for general, not necessarily symmetric data, but let's be modest for now. So far all analytical and numerical results support the conjecture that the above is indeed true and that in particular the spherically symmetric, asymptotically flat Einstein-Vlasov system satisfies the weak cosmic censorship conjecture. An existence result for singularity-free solutions for restricted, small data has been known for some time and is reviewed in the next section. A class of data which lead to gravitational collapse as described above has been established more recently, and this result is discussed in Section 4. 
3. Local and global existence results. In this section we review a number of results from the literature for the spherically symmetric, asymptotically flat EinsteinVlasov system. They serve as either background or counterparts to the results on gravitational collapse, which we state and discuss in the next section.

3.1. Local existence and continuation. Due to our choice of $v$ as a noncanonical momentum variable, the system (2.9)-(2.17) has the following nice feature. If a distribution function $f$ is given, then the source terms $\rho, p, j$, and $q$ can be computed from it without reference to the metric. Given $\rho$ and observing the boundary condition (2.8) the field equation (2.10) can be integrated to yield

$$
e^{-2 \lambda}=1-\frac{2 m}{r}
$$

where the quasi-local mass $m$ is given by

$$
m(t, r):=4 \pi \int_{0}^{r} \rho(t, \eta) \eta^{2} d \eta
$$

Given $p$ and $\lambda$ the field equation (2.11) together with the boundary condition (2.7) determines $\mu$ :

$$
\mu(t, r)=\exp \left(-\int_{r}^{\infty} e^{2 \lambda(t, \eta)}\left(\frac{m(t, \eta)}{\eta^{2}}+4 \pi \eta p(t, \eta)\right) d \eta\right) .
$$

Finally, if both $\lambda$ and $\mu$ are given and sufficiently regular, then $f$ is determined from its initial data by the method of characteristics:

$$
f(t, x, v)=\stackrel{\circ}{f}((X, V)(0, t, x, v)),
$$

where $(X, V)(s, t, x, v)$ is the solution of the characteristic system

$$
\dot{x}=e^{\mu-\lambda} \frac{v}{\sqrt{1+|v|^{2}}}, \dot{v}=-\left(\partial_{t} \lambda \frac{x \cdot v}{r}+e^{\mu-\lambda} \partial_{r} \mu \sqrt{1+|v|^{2}}\right) \frac{x}{r}
$$

of the Vlasov equation (2.9) satisfying $(X, V)(t, t, x, v)=(x, v)$, and $\stackrel{\circ}{f}$ is the prescribed data at time $t=0$. Notice that the characteristics are now parameterized by coordinate time instead of proper time as in (2.2).

The iterative scheme indicated above can be used to prove a local existence and uniqueness theorem together with an extension criterion.

Theorem 3.1. Let $\stackrel{\circ}{f} \in C_{c}^{1}\left(\mathbb{R}^{6}\right)$ be nonnegative, spherically symmetric, and such that for the induced quasi-local mass,

$$
2 \stackrel{\circ}{m}(r) / r<1, r>0 .
$$

Then there exists a unique regular solution $f$ of the asymptotically flat, spherically symmetric Einstein-Vlasov system with $f(0)=\stackrel{\circ}{f}$ on a maximal interval of existence $[0, T[$ with $T>0$. If

$$
\sup \{|v| \mid(t, x, v) \in \operatorname{supp} f, 0 \leq t<T\}<\infty,
$$

then $T=\infty$. 
This result was first established in [39; see also 37. Some comments are in order.

Functions in $C_{c}^{1}\left(\mathbb{R}^{6}\right)$ are by definition continuously differentiable and compactly supported. A solution is called regular if the derivatives which appear in the system exist in the classical sense and are continuous; for the precise definition, we refer to [37.

The restriction (3.6) on the initial data is necessary in view of (3.1) because that equation defines $\lambda$ only as long as $2 m(t, r) / r<1$, so we have to require this for the initial data. This is related to the fact that, as noted in the first section, Schwarzschild coordinates cannot cover regions of spacetime which contain trapped surfaces.

A local existence and uniqueness result for the Einstein-Vlasov system without a symmetry assumption was established by Y. Choquet-Bruhat [12]. However, in order to extend a local solution to a global one based on the latter result, one would have to control high-order Sobolev norms of the solution. The extension criterion provided in Theorem 3.1 is much less demanding and forms a more convenient starting point for investigating global properties of solutions.

The major simplification of the system due to the symmetry assumption is the fact that for given source terms the metric is completely determined by the two constraint equations (2.10) and (2.11) which are ordinary differential equations in $r$. The metric has no independent degrees of freedom, and this rules out gravitational radiation, much like the Vlasov-Maxwell system being reduced to the Vlasov-Poisson system if spherical symmetry is assumed. But if $\lambda$ is defined by (3.1), then it becomes rather unpleasant to control $\partial_{t} \lambda$ which appears in the Vlasov equation and to make sure that the condition $2 m(t, r) / r<1$ is preserved. In 37, this was resolved by considering first a modified system where (2.13) was left out and $\partial_{t} \lambda$ was replaced by a quantity $\tilde{\lambda}$ defined by (2.12). Then one can show a posteriori that indeed $\partial_{t} \lambda=\tilde{\lambda}$ and that (2.13) holds as well. Equation (2.12) can then be used to control $\partial_{t} \lambda$ and therefore $\lambda$ in terms of

$$
P(t):=\sup \{|v| \mid(s, x, v) \in \operatorname{supp} f, 0 \leq s \leq t\} ;
$$

notice that by (3.4) the function $f(t)$ is bounded so that the source terms $\rho, p, j, q$ are bounded by powers of $P$.

Equation (2.13) also has its role to play. To establish the convergence of the iterative scheme one needs uniform bounds on certain second-order derivatives of the metric coefficients. But since in (2.10) $-(2.12)$ the first-order derivatives depend in a pointwise way on the source terms, it seems that one needs to control derivatives of these source terms. In the corresponding results for the Vlasov-Poisson or Vlasov-Maxwell systems one exploits the fact that the field quantities depend on the source terms through spatial or spacetime integrals. The corresponding field equations are smoothing; cf. [10, 26, 38]. Here the field equation (2.13) provides this smoothing effect, because it turns out that in order to control the derivatives of the characteristic flow with respect to the initial data only a certain combination of second-order derivatives of the metric coefficients is needed, and this combination is precisely the one which appears in (2.13) and is therefore controlled by $q$.

In the context of these arguments and also in what follows some further information is useful. By (2.10) and (2.11),

$$
\partial_{r} \mu+\partial_{r} \lambda \geq 0
$$


and together with the boundary condition (2.7) and (3.1), this implies that

$$
\mu-\lambda \leq \mu+\lambda \leq 0 \text {. }
$$

Solutions of the Einstein-Vlasov system satisfy the following conservation laws. The Vlasov equation implies that the number of particles

$$
N=\iint e^{\lambda(t, r)} f(t, x, v) d v d x
$$

is conserved. More importantly, if we observe that $j$ is for each fixed time $t$ compactly supported so that by (2.12),$\partial_{t} \lambda(t, r)=0$ for $r$ sufficiently large, we conclude from (3.1) that the ADM mass

$$
M=\lim _{r \rightarrow \infty} m(t, r)=\iint \sqrt{1+|v|^{2}} f(t, x, v) d v d x
$$

is conserved.

3.2. Global existence for small data. A natural question when investigating a nonlinear system of PDEs is whether small initial data lead to global solutions which disperse. As mentioned above, no such smallness condition can be formulated for the at least formally closely related Einstein-dust system, cf. [13, but for true Vlasov matter such a global result for small data does exist.

Theorem 3.2. For all $R>0$ there exists $\epsilon>0$ such that if $f$ is a maximal solution of the asymptotically flat, spherically symmetric Einstein-Vlasov system with $f(0)=\stackrel{\circ}{f}$ satisfying

$$
\stackrel{\circ}{f}(x, v)=0 \text { for }|x|+|v|>R
$$

and

$$
\|\stackrel{\circ}{\|}\|_{\infty}<\epsilon,
$$

then the solution exists globally in $t$. Moreover, the solution disperses in the sense that

$$
\|\rho(t)\|_{\infty} \leq C(1+|t|)^{-3}, t \in \mathbb{R},
$$

and the spacetime is geodesically complete.

This result was first proven in [39]; see also [37, 47]. Similar results were known both for the Vlasov-Poisson and the Vlasov-Maxwell system; cf. [9, 27, 38]. The basic dispersive mechanism in the system can be seen as follows. By (3.4) and the change of variables formula,

$$
\begin{aligned}
\rho(t, x) & =\int \sqrt{1+|v|^{2}} \stackrel{\circ}{f}((X, V)(0, t, x, v)) d v \\
& =\int \sqrt{1+|v|^{2}} \stackrel{\circ}{f}(X, V(0, t, x, v))\left|\operatorname{det}\left(\frac{\partial X(0, t, x, v)}{\partial v}\right)\right|^{-1} d X .
\end{aligned}
$$

Now in the flat Minkowski case, $\lambda=\mu=0, X(0, t, x, v)=x-t v / \sqrt{1+|v|^{2}}$, and the determinant above grows like $t^{3}$. If the field terms satisfy suitable decay conditions, this turns out to remain correct. The decay of $\rho$ and the other source terms in turn implies decay for the field terms, and a bootstrap argument gives the result. Here it again becomes important that a certain combination of second-order derivatives of the metric coefficients can be controlled via the source terms by the field equation (2.13). 
The above argument proves that the solution is global with respect to the chosen time coordinate. However, this does not automatically imply that the maximal Cauchy development of the corresponding initial data is singularity-free. By definition, a spacetime contains a singularity if there exists a timelike or null geodesic, i.e., a world line of a particle or a photon, whose maximally extended domain of affine parameter, i.e., proper time in the case of a particle, is not the whole real line. The geometrically invariant, coordinate-free characterization of a singularity-free or global spacetime is therefore that all timelike and null geodesics exist on the whole real line. In the course of the proof of the above bootstrap argument, one obtains sufficient decay information on the Christoffel symbols to conclude that indeed all maximally extended geodesics in this spacetime are complete, i.e., exist on the whole real line.

A mechanism which also leads to global existence but is different from dispersion induced by small data is that initially all the particles move outward sufficiently fast to prevent recollapse; cf. [5]. For a collisionless gas of massless particles a result analogous to Theorem 3.2 has been shown in 22 .

3.3. Possible breakdown must occur at the center first. The next question is whether large data lead to a breakdown of the solution. It is important to realize that the fact that, as shown in the next section, certain data do lead to gravitational collapse and the formation of black holes does certainly not rule out the possibility that in Schwarzschild coordinates all solutions of the spherically symmetric, asymptotically flat Einstein-Vlasov system are global. If the weak cosmic censorship conjecture holds for this system, then, physically speaking, an observer who is very far away (ideally at spatial infinity) should not be able to observe the singularity so that for him the universe should look singularityfree, and in this context one should note that Schwarzschild time asymptotically coincides with the proper time measured by such an observer. So far, all numerical simulations and analytical results support the conjecture that all solutions are global in Schwarzschild time. In all cases where a gravitational collapse was simulated numerically the solution did not seem to break down in finite Schwarzschild time and an event horizon did form; cf. 7, 31, 44. In the next section we discuss a recent analytical result which shows that, at least for a certain class of data, solutions do again exist globally in Schwarzschild time, but they do undergo a gravitational collapse and form a black hole. In the context of that result it is important to know that if a solution should develop a singularity in finite Schwarzschild time, then this must happen at the center first.

THEOREM 3.3. Let $f$ be a regular solution of the spherically symmetric, asymptotically flat Einstein-Vlasov system on a time interval $[0, T[$. Suppose that there exists an open neighborhood $U$ of the point $(T, 0)$ such that

$$
\sup \left\{|v| \mid(t, x, v) \in \operatorname{supp} f \cap\left(U \times \mathbb{R}^{3}\right)\right\}<\infty .
$$

Then $f$ extends to a regular solution on $\left[0, T^{\prime}\right.$ for some $T^{\prime}>T$.

This theorem was established in [43]. In order to explain the mechanism behind this, we introduce coordinates on the mass shell which are adapted to the symmetry:

$$
r=|x|, w=\frac{x \cdot v}{r}, L=|x \times v|^{2} .
$$


Spherical symmetry of $f$ implies that, by abuse of notation,

$$
f(t, x, v)=f(t, r, w, L) .
$$

One should think of $w$ as the noncanonical radial momentum and $L$ as the modulus of angular momentum squared of a particle. Due to spherical symmetry, $L$ is conserved along characteristics, and the characteristic system written in $(r, w, L)$ takes the form

$$
\begin{aligned}
\dot{r} & =e^{\mu-\lambda} \frac{w}{E}, \\
\dot{w} & =-\left(\partial_{t} \lambda w+e^{\mu-\lambda} \partial_{r} \mu E-e^{\mu-\lambda} \frac{L}{r^{3} E}\right), \\
\dot{L} & =0,
\end{aligned}
$$

where

$$
E=E(r, w, L):=\sqrt{1+w^{2}+L / r^{2}}=e^{\mu} p^{0} .
$$

The source terms take the form

$$
\begin{aligned}
\rho(t, r) & =\frac{\pi}{r^{2}} \int_{-\infty}^{\infty} \int_{0}^{\infty} E f(t, r, w, L) d L d w, \\
p(t, r) & =\frac{\pi}{r^{2}} \int_{-\infty}^{\infty} \int_{0}^{\infty} \frac{w^{2}}{E} f(t, r, w, L) d L d w, \\
j(t, r) & =\frac{\pi}{r^{2}} \int_{-\infty}^{\infty} \int_{0}^{\infty} w f(t, r, w, L) d L d w ;
\end{aligned}
$$

the quantity $q$ is not needed in what follows. Since on the support of $f$ the quantity $L$ is bounded initially, it remains bounded for all time. In order to establish the theorem one needs to control the increase in $|v|$ along characteristics which stay away from the center. Because of the relation $|v|^{2}=w^{2}+L / r^{2}$, it is sufficient to control $w$ along such a characteristic. If we substitute the expressions for $\partial_{t} \lambda$ and $\partial_{r} \mu$ and for the source terms into (3.12), we find that

$$
\begin{aligned}
\dot{w}= & e^{\mu-\lambda} \frac{L}{r^{3} E}-e^{\mu+\lambda} \frac{m(s, r)}{r^{2}} E \\
& +4 \pi r e^{\mu+\lambda} \frac{\pi}{r^{2}} \int_{-\infty}^{\infty} \int_{0}^{\infty}\left(w \tilde{w}-E \frac{\tilde{w}^{2}}{\tilde{E}}\right) f(s, r, \tilde{w}, \tilde{L}) d \tilde{L} d \tilde{w}
\end{aligned}
$$

with the obvious definition of $\tilde{E}$. Now $L$ is bounded, $r$ is bounded away from zero, $m$ is bounded by $M$, and $\mu-\lambda \leq \mu+\lambda \leq 0$ by (3.7). Hence the term to focus on is the double integral. But

$$
\begin{aligned}
w \tilde{w}-E \frac{\tilde{w}^{2}}{\tilde{E}} & =\frac{\tilde{w}}{\tilde{E}} \frac{w^{2}\left(1+\tilde{w}^{2}+\tilde{L} / r^{2}\right)-\tilde{w}^{2}\left(1+w^{2}+L / r^{2}\right)}{w \tilde{E}+\tilde{w} E} \\
& =\frac{\tilde{w}}{\tilde{E}} \frac{w^{2}\left(1+\tilde{L} / r^{2}\right)-\tilde{w}^{2}\left(1+L / r^{2}\right)}{w \tilde{E}+\tilde{w} E},
\end{aligned}
$$

provided the denominator does not vanish. In the last step the term $w^{2} \tilde{w}^{2}$, which is the worst one concerning powers of $w$ or $\tilde{w}$, canceled, and this is the crucial observation in 
order to establish Theorem 3.3 , since it leads to an estimate of the form

$$
P(t) \leq P(0)+C \int_{0}^{t} P(s) \ln P(s) d s
$$

cf. the continuation criterion in Theorem 3.1 .

The result above refers to the Einstein-Vlasov system written in Schwarzschild coordinates. The analogous result in so-called maximal-isotropic coordinates was established in [47. In 23] the analogous result is established using coordinates which cover the whole spacetime, even in the presence of trapped surfaces.

To appreciate this result it is instructive to think of a spherically symmetric solution of the Einstein-dust system. In that system all particles move radially, and particles at the same radius $r$ have the same momentum, i.e., they remain on the same sphere which can grow or shrink as a whole; cf. (2.6). If two such spheres cross, the system experiences a so-called shell-crossing singularity which occurs at some positive radius. If one wants to study the formation of event horizons and spacetime singularities in the Einstein-dust system, one has to handle the problem that the solution may break down due to a shell-crossing singularity before the objects of interest have actually evolved.

4. Gravitational collapse and formation of black holes. As pointed out repeatedly above, Schwarzschild coordinates do not cover regions of spacetime which contain trapped surfaces. Since in gravitational collapse the latter typically appear before a singularity forms, one may well argue that in order to investigate gravitational collapse and the formation of black holes, one should better not use these coordinates to begin with. On the other hand, they do have advantages from an analysis point of view, and one may still hope to derive the desired information on gravitational collapse from the asymptotic behavior of the solution for large Schwarzschild time. But for large data which possibly lead to gravitational collapse there is so far no global existence result in Schwarzschild time. In order to bypass this difficulty we study the solutions in a coordinate region which avoids the center, on which we can consistently formulate and study the corresponding Cauchy problem, and which is large enough to allow us to conclude the formation of black holes. That the behavior of the solution on this coordinate region indeed implies the weak cosmic censorship conjecture and the formation of a black hole is shown in Section 4.3, where we rely on a mathematical formulation of the corresponding concepts given in [20.

4.1. The system on an outer domain. We study the solutions to the spherically symmetric, asymptotically flat Einstein-Vlasov system (2.9)-(2.17) on the exterior region

$$
D:=\left\{( t , r ) \in \left[0, \infty\left[^{2} \mid r \geq \gamma^{+}(t)\right\},\right.\right.
$$

where $\gamma^{+}$is an outgoing radial null geodesic originating from some $r=r_{0}>0$, i.e.,

$$
\frac{d \gamma^{+}}{d s}(s)=e^{(\mu-\lambda)\left(s, \gamma^{+}(s)\right)}, \gamma^{+}(0)=r_{0} .
$$

This equation is obtained from (3.11) by replacing the 1 in the definition of $E$ by 0 , i.e., the particle under consideration has rest mass 0 like a photon should, and by putting $L=0$, i.e., the photon moves radially. That the photon is outgoing, i.e., moving away 
from the center, means that $w>0$, and hence $w / E=1$. In order to restrict the analysis of the system to the region $D$ we have to find a replacement for the boundary condition (2.8). We prescribe the total ADM mass $M>0$ and redefine the quasi-local mass by

$$
m(t, r)=M-4 \pi \int_{r}^{\infty} \rho(t, \eta) \eta^{2} d \eta
$$

while retaining the definition (3.1) for $\lambda$. Clearly, a solution of the system as considered in the previous section, when restricted to $D$, is a solution of this modified system. Moreover, characteristics of the Vlasov equation can pass from the region $D$ into the region $\left\{r<\gamma^{+}(t)\right\}$ but not the other way around so that initial data $\stackrel{\circ}{f}$ posed for $r>r_{0}$ completely determine the solution on the outer domain $D$. Such data need to satisfy the restrictions (3.6) and

$$
M_{\mathrm{out}}=4 \pi \int_{r_{0}}^{\infty} \stackrel{\rho}{\rho}(\eta) \eta^{2} d \eta<M
$$

where $\stackrel{\circ}{\rho}$ is induced by $\stackrel{\circ}{f}$. Then $\lim _{r \rightarrow \infty} m(t, r)=M$ and $0 \leq m \leq M$. The crucial question is whether one can specify data such that $\gamma^{+}$has the property that

$$
\lim _{s \rightarrow \infty} \gamma^{+}(s)<\infty
$$

While this is not sufficient to conclude the formation of a black hole, it turns out to be the main step towards that goal.

It is important to note that the behavior or even the nature of the matter in the region $\left\{r<\gamma^{+}(t)\right\}$ is not going to be relevant in what follows. For example one can equally well think of Vlasov data being posed on $\{r \geq 0\}$, but only the data on $\left\{r \geq r_{0}\right\}$ need to be properly restricted in order to obtain the desired behavior of the solution on the outer domain $D$. What is essential is that there initially is and hence remains some matter in the region $\left\{r<\gamma^{+}(t)\right\}$ as guaranteed by the condition $M_{\text {out }}<M$.

4.2. The main result-analysis in Schwarzschild coordinates. The initial data $\stackrel{\circ}{f} \in$ $C_{c}^{1}\left(\mathbb{R}^{6}\right)$ for the outer matter should satisfy the condition that on $\operatorname{supp} \stackrel{\circ}{f}$

$$
R_{0} \leq r \leq R_{1}, w \leq W_{-},
$$

where we use the variables $(r, w, L)$ introduced above, and

$$
0<r_{0}<R_{0}<R_{1}, W_{-}<0 .
$$

In particular, all particles move inward initially. These parameters can be specified in such a way that the data are close to violating the condition $2 m^{\circ}(r) / r<1$, that the particles continue to move inward on the outer domain $D$, and that (4.5) holds. The main result is the following.

TheOREm 4.1. There exists a class of regular initial data for the spherically symmetric Einstein-Vlasov system such that for such data the corresponding solution exists on $D$, and

$$
\lim _{s \rightarrow \infty} \gamma^{+}(s)<\infty
$$

In addition the following holds: 
(a) For $t \rightarrow \infty$ no matter remains in the region $\{r>2 M\}$. More precisely, the solution is vacuum and the metric equals the Schwarzschild metric (1.1) with mass $M$ in the region

$$
t \geq 0 \text { and } r \geq 2 M+\alpha e^{-\beta t}
$$

Here $\alpha, \beta>0$ depend only on the initial data class.

(b) In the outer region $D$ and for $r \leq 2 M$,

$$
\lim _{t \rightarrow \infty} \mu(t, r)=-\infty
$$

more precisely,

$$
\mu(t, r) \leq \ln \left(\frac{\alpha e^{-\beta t}}{2 M+\alpha e^{-\beta t}}\right)^{1 / 2}
$$

for all $t \geq 0$ and $\gamma^{+}(t) \leq r \leq 2 M+\alpha e^{-\beta t}$. This implies that for $c \leq 2 M$ the timelike lines $r=c$ are incomplete; i.e., they have finite proper length, and this length is uniformly bounded.

(c) The radially outgoing null geodesic which does not escape to infinity and is furthest to the right with this property gets trapped precisely at the Schwarzschild radius $r=2 M$. More precisely, we define

$$
\begin{aligned}
r^{*}:=\sup \left\{r \geq r_{0} \quad \mid\right. & \text { the radially outgoing null geodesic } \gamma \text { with } \\
& \left.\gamma(0)=r \text { satisfies } \lim _{s \rightarrow \infty} \gamma(s)<\infty\right\},
\end{aligned}
$$

and we let $\gamma^{*}$ be the radially outgoing null geodesic with $\gamma^{*}(0)=r^{*}$. Then

$$
\lim _{s \rightarrow \infty} \gamma^{*}(s)=2 M
$$

and every radially outgoing null geodesic $\gamma$ with $\gamma(0)>r^{*}$ is future complete; i.e., it exists on $\left[0, \infty\left[\right.\right.$ in an affine parameterization, and $\lim _{s \rightarrow \infty} \gamma(s)=\infty$.

If a solution with mass $M$ of the spherically symmetric, asymptotically flat EinsteinVlasov system collapses to a black hole of mass $M$ and if we coordinatize this solution by Schwarzschild coordinates, then the asymptotic behavior as $t \rightarrow \infty$ of this solution should be as given by this theorem. In the next subsection we show that this behavior actually implies that a black hole forms in the sense of a suitable, coordinate-independent formulation of this concept.

For a complete proof of Theorem 4.1 we refer to [6]. Here we want to highlight some central arguments. The first of these makes sure that the particles in the outer domain $D$ keep moving inward in a controlled way. Since initially $w \leq W_{-}<0$ for all particles, this remains true on some time interval. On this time interval and along any characteristic 
in $\operatorname{supp} f$,

$$
\begin{aligned}
\frac{d}{d s}\left(e^{-\lambda} w\right)= & -\frac{4 \pi^{2}}{r} e^{\mu} \int_{-\infty}^{\infty} \int_{0}^{\infty}\left[\sqrt{\frac{\tilde{E}}{E}} w-\sqrt{\frac{E}{\tilde{E}}} \tilde{w}\right]^{2} f d \tilde{L} d \tilde{w} \\
& -e^{\mu} \frac{m}{r^{2}}\left(\frac{1+L / r^{2}}{E}+\frac{2 L}{r^{2} E}\right)+e^{\mu} \frac{L}{r^{3} E} \\
\leq & -e^{\mu} \frac{m}{r^{2}}\left(\frac{1+L / r^{2}}{E}+\frac{2 L}{r^{2} E}\right)+e^{\mu} \frac{L}{r^{3} E} .
\end{aligned}
$$

Differentiating (3.1) w.r.t. $t$ and using (2.12) leads to $\partial_{t} m=-4 \pi r^{2} e^{\mu-\lambda} j \geq 0$ on the time interval we consider. It follows that $m(s, r) \geq m(0, r)=\stackrel{\circ}{m}(r)$. Thus as long as the characteristic remains in $D$,

$$
\frac{d}{d s}\left(e^{-\lambda} w\right) \leq e^{\mu} \frac{1}{r^{3} E}\left(L-\frac{3 L}{r} \stackrel{\circ}{m}(r)-r \stackrel{\circ}{m}(r)\right) .
$$

We require that

$$
0<L<\frac{3 L}{r} \stackrel{\circ}{m}(r)+r \stackrel{\circ}{m}(r), r \in\left[r_{0}, R_{1}\right]
$$

on supp $\stackrel{\circ}{f}$. Then the above estimate together with a bootstrap argument and the fact that $e^{\lambda} \geq 1$ shows that

$$
w \leq\left(\min _{r \in\left[R_{0}, R_{1}\right]} e^{-\lambda(0, r)}\right) W_{-}
$$

on $\operatorname{supp} f \cap D$.

Before going further, some comments on the condition (4.6) are in order. By our general setup, some mass must be inside $\left\{r \leq r_{0}\right\}$ initially, and this mass is a lower bound on $\stackrel{\circ}{m}$ in (4.6). The role of this mass is not to pull the particles inward, but to keep them focused towards the center. The smaller their angular momentum is, i.e., the better they are aimed straight towards the center, the smaller can the central mass be chosen initially. Notice that for spherically symmetric dust, which is used as a matter model in [32] and [13, all particles have angular momentum equal to zero.

The second issue in the proof of Theorem 4.1 we want to touch upon is the limiting behavior of $\gamma^{+}$. The basic idea is to consider a radially ingoing null geodesic $\gamma^{-}$which starts to the left of the outer matter and to the right of $\gamma^{+}$, i.e.,

$$
\frac{d \gamma^{-}}{d s}(s)=-e^{(\mu-\lambda)\left(s, \gamma^{-}(s)\right)}, \quad r_{0}<r_{1}=\gamma^{-}(0)<R_{0} .
$$

Then initially and therefore as long as $\gamma^{+}$and $\gamma^{-}$do not intersect, there is no matter in the region between the outgoing and the ingoing null geodesic. This fact can be used to estimate their relative velocity in such a way that in Schwarzschild time they actually never intersect. This proves the limiting behavior of $\gamma^{+}$and furthermore shows that the matter which is initially in $D$ stays strictly to the right of $\gamma^{+}$and therefore in $D$ for all future Schwarzschild time. In order to estimate how far the two null geodesics can move, at most we observe that by (3.3),

$$
\mu(t, r) \leq-\int_{r}^{\infty} e^{2 \lambda(t, s)} \frac{m(t, s)}{s^{2}} d s=: \hat{\mu}(t, r),
$$


and hence

$$
\left|\dot{\gamma}^{ \pm}\right| \leq e^{\hat{\mu}}
$$

We wish to see that the right-hand side becomes small, and to this end we observe that the following chain of estimates yields a lower bound for $\partial_{t} \hat{\mu}$ :

$$
\begin{aligned}
1-e^{(\mu+\lambda)(t, r)} & =\int_{r}^{\infty}\left(\partial_{r} \lambda+\partial_{r} \mu\right)(t, \eta) e^{\mu+\lambda} d \eta \\
& =4 \pi \int_{r}^{\infty} \eta(\rho+p)(t, \eta) e^{\mu+\lambda} e^{2 \lambda} d \eta \\
& \leq 4 \pi \int_{r}^{\infty} 3 \eta(-j(t, \eta)) e^{\mu+\lambda} e^{2 \lambda} d \eta \\
& =-3 \int_{r}^{\infty} \eta \frac{\partial}{\partial t}\left(e^{2 \lambda} \frac{m(t, \eta)}{\eta^{2}}\right) d \eta \\
& \leq-3 R_{1} \partial_{t} \hat{\mu}(t, r) .
\end{aligned}
$$

In the first estimate we exploit the fact that not only is $j$ negative, but by choosing $\left|W_{-}\right|$ large the source terms $\rho$ and $p$ can be estimated by a suitable multiple of $-j$.

If one puts all the details which are left out here together, one obtains a list of conditions on the initial data which make sure that these estimates hold true for all future Schwarzschild time. The resulting conditions are the following:

$$
\left.\left.\operatorname{supp} \stackrel{\circ}{f} \subset\left[R_{0}, R_{1}\right] \times\right]-\infty, W_{0}\right] \times[0, \infty[
$$

with

$$
\begin{aligned}
0<r_{0}<r_{1} & =2 M<R_{0}=\frac{r_{1}+R_{1}}{2}<R_{1}, W_{-}<0, \\
0 & <\stackrel{\circ}{m}\left(r_{0}\right)<M, \quad \frac{\stackrel{\circ}{m}\left(r_{0}\right)}{r_{0}}<\frac{8}{9}
\end{aligned}
$$

and either

or

$$
R_{1}-r_{1}<\frac{r_{1}-r_{0}}{6}
$$

$$
\sqrt{1-\frac{r_{1}}{R_{1}}}<\min \left\{\frac{1}{6}, \frac{r_{0}^{2}}{36 R_{1} M}, \frac{r_{1}-r_{0}}{24 R_{1}}\right\} .
$$

Since $r_{1}=2 M$, the latter two conditions both say that $2 M / R_{1}$ must be close to 1 . Once $r_{0}, r_{1}, R_{0}, R_{1}$, and $\stackrel{\circ}{m}\left(r_{0}\right)$ have been chosen, $\left|W_{-}\right|$has to be chosen sufficiently large where we refrain from making this precise here. Any initial distribution on $\left\{r>r_{0}\right\}$ which satisfies (3.6) and (4.6) is admissible in Theorem 4.1, and it is easy to see that there exist such data.

Since the various parameters which enter the definition of our class of admissible data are defined in terms of inequalities, the set of data has "nonempty interior", in the sense that sufficiently small perturbations of initial data in the "interior" of this set belong to it as well.

The crucial step in the proof of the remainder of Theorem 4.1 is to show that all particles move towards $r=2 M$ with the stated estimate. As a first step note that by 
(3.1) and (3.3),

$$
(\mu-\lambda)(t, r) \geq \ln \frac{r-2 M}{r}, r>2 M
$$

Together with the control for the radial momentum of the particles this implies that along any characteristic in the matter support,

$$
\dot{r}=e^{(\mu-\lambda)(s, r)} \frac{w}{E} \leq-C e^{(\mu-\lambda)(s, r)} \leq-C \frac{r-2 M}{r}
$$

as long as $r>2 M ; C>0$ is determined by the initial data parameters. Integrating this differential inequality proves the support estimate in the theorem.

The spherically symmetric, asymptotically flat Einstein-Vlasov system has a wide variety of static solutions with finite ADM mass and compact matter support; cf. 36, 41, 42. Particularly interesting examples of initial data to which our results apply are obtained if the matter for $r \leq r_{0}$ is represented by such a static solution.

COROLlary 4.2. Let $f_{s}$ be a static solution of the spherically symmetric, asymptotically flat Einstein-Vlasov system with finite ADM mass $M_{s}>0$ and finite radius $r_{s}>0$. Define $r_{0}=r_{s}$, let $r_{1}>r_{0}$ be arbitrary, $M=r_{1} / 2$, and $M_{\text {out }}=M-M_{s}$; the latter quantity is positive. Then one can construct data $f$ on $\left\{r \geq r_{s}\right\}$ such that the solution of the Einstein-Vlasov system launched by $f_{s}+\stackrel{\circ}{f}$ has the properties stated in Theorem 4.1 , it exists for all $t \geq 0$ and $r \geq 0$, and it coincides with the static solution $f_{s}$ for all $r \leq \gamma^{+}(t)$ and $t \geq 0$.

It is at this point that the choice $8 / 9$ in (4.9) is relevant; for the proof of Theorem4.1, any positive constant less than 1 would do. But in [4] it is shown that for any steady state of the spherically symmetric Einstein-Vlasov system the condition $2 m(r) / r<8 / 9$ holds for all $r>0$, and this bound is actually sharp; cf. [3].

Given the fact that the solutions described in Theorem 4.1 undergo gravitational collapse and form a black hole it may seem strange that in the center of such a solution a steady state comfortably sits for all $t \geq 0$. But this is of course due to the fact that $t$ is Schwarzschild time. In the region $r<2 M$ the solution can be extended using a different time coordinate, and if the latter is properly chosen, then the outer matter will all pass within $r<2 M$, it will crash into the steady state, and all the matter will finally collapse into a spacetime singularity. To support all phases of this evolution with rigorous theorems is one of our projects for current and future research; cf. Section 5.3.

On the other hand, the fact that the steady state sits undisturbed in the center as long as none of the outer matter reaches it is easily understood from a physics point of view, and just as easily proven. In spherical symmetry, particle orbits within a certain radius $r$ are not influenced by matter which is at strictly larger radii. Hence adding the outer matter shell does not change the fact that the steady state satisfies the Einstein-Vlasov system on $\left\{r \leq r_{0}\right\}$ as long as the outer matter remains outside that region.

4.3. Weak cosmic censorship and the formation of a black hole. The result of the previous section shows in particular that no particle and no light ray can escape from the region $\left\{r \leq \gamma^{*}(t)\right\} \subset\{r \leq 2 M\}$. Since outside of this domain the solution is global, this means in particular that no causal curve originating in a possible singularity can reach the region $\{r>2 M\}$. However, all this refers to the formulation of the Einstein-Vlasov 
system in Schwarzschild coordinates, and we are not (yet) allowed to conclude that our spacetime satisfies the weak cosmic censorship conjecture and that a black hole forms. In the present subsection, we address these questions in a coordinate-independent manner. We start with showing that spacetimes as obtained in the previous subsection satisfy the weak cosmic censorship conjecture which in heuristic terms says that no singularities can be observed from infinity even if the observations are allowed to continue indefinitely. Expressed more precisely we have to show:

Proposition 4.3. The spacetimes obtained in Theorem 4.1 possess a complete future null infinity.

The concept of future null infinity usually refers to a conformal compactification of the spacetime under investigation which attaches a boundary at infinity; cf. [51, 11.1]. We prefer to follow Christodoulou [20, p. A26] for the definition of the term "possess a complete future null infinity".

The set $B_{0}:=\left\{(0, r) \mid r<R_{1}\right\}$ has compact closure, and the boundary $C_{0}^{+}$of its causal future is given by the radially outgoing null geodesic $\gamma_{1}$ starting at $R_{1}$; we recall that $R_{1}$ is the outer radius of the initial matter support in Theorem 4.1. By that theorem, $\gamma_{1}$ is future complete. Consider now a domain $B:=\left\{(0, r) \mid r<R_{2}\right\}$ with $R_{2}>R_{1}$ and the boundary $C^{-}$of its domain of dependence which is given by the radially ingoing null geodesic $\gamma_{2}$ starting at $R_{2}$. According to the definition in 20, we must show that the affine length of $\gamma_{2}$, measured from the intersection of $\gamma_{1}$ and $\gamma_{2}$, goes to infinity as $R_{2} \rightarrow \infty$. In doing so the affine parameterization of $\gamma_{2}$ must be normalized in such a way that its tangent at $\left(0, R_{2}\right)$ equals the vector $T-N$, where $T$ is the future directed unit normal to the initial hypersurface $\{t=0\}$, i.e., $T=\left(e^{-\mu\left(0, R_{2}\right)}, 0,0,0\right)$, and $N$ is the outward unit normal to $B$ in the initial hypersurface, i.e., $N=\left(0, e^{-\lambda\left(0, R_{2}\right)}, 0,0\right)$.

It turns out that already the affine length of $\gamma_{2}$ between the intersections with $\gamma_{1}$ and with the line $r=R_{1}$ goes to infinity as $R_{2} \rightarrow \infty$. But on the region $\left\{r \geq R_{1}\right\}$ we have according to Theorem 4.1, vacuum with metric given by

$$
e^{2 \mu(t, r)}=1-\frac{2 M}{r}=e^{-2 \lambda(t, r)} .
$$

When parameterized by coordinate time, radial null geodesics in the region $\left\{r \geq R_{1}\right\}$ satisfy the estimates

$$
1-\frac{2 M}{R_{1}} \leq 1-\frac{2 M}{r}=e^{\mu-\lambda}=\left|\frac{d r}{d t}\right| \leq 1 .
$$

Let $\left(T^{*}, R^{*}\right)$ denote the point where $\gamma_{1}$ and $\gamma_{2}$ intersect. By the above estimate, $\gamma_{1}(t) \leq$ $R_{1}+t$ and $\gamma_{2}(t) \geq R_{2}-t$ which implies that $T^{*} \geq\left(R_{2}-R_{1}\right) / 2$ and hence

$$
R^{*} \geq R_{1}+\frac{R_{2}-R_{1}}{2}\left(1-\frac{2 M}{R_{1}}\right)
$$

Consider now an affine parameterization $\tau \mapsto(t, r, \theta, \varphi)(\tau)$ of $\gamma_{2}, \tau \geq 0$, with $(t, r)(0)=$ $\left(0, R_{2}\right)$. Since $\gamma_{2}$ is radial, $\theta=\pi / 2, \varphi=0$. Since $\gamma_{2}$ is null,

$$
-e^{2 \mu}\left(\frac{d t}{d \tau}\right)^{2}+e^{2 \lambda}\left(\frac{d r}{d \tau}\right)^{2}=0,
$$


and since $\gamma_{2}$ is ingoing, i.e., $d r / d \tau<0$, we find that

$$
\frac{d t}{d \tau}=-e^{\lambda-\mu} \frac{d r}{d \tau}
$$

By the geodesic equation,

$$
\begin{aligned}
\frac{d^{2} r}{d \tau^{2}} & =-e^{2(\mu-\lambda)} \partial_{r} \mu\left(\frac{d t}{d \tau}\right)^{2}-\partial_{r} \lambda\left(\frac{d r}{d \tau}\right)^{2}-2 \partial_{t} \lambda \frac{d t}{d \tau} \frac{d r}{d \tau} \\
& =4 \pi r e^{2 \lambda}\left(\frac{d r}{d \tau}\right)^{2}[-p-\rho-2 j]=0
\end{aligned}
$$

i.e., $d r / d \tau=$ const $=: \sigma$ as long as $\gamma_{2}$ is in the vacuum region $\left\{r \geq R_{1}\right\}$. The normalization condition mentioned above requires that

$$
\left(\frac{d t}{d \tau}, \frac{d r}{d \tau}\right)(0)=\left(-e^{\lambda\left(0, R_{2}\right)-\mu\left(0, R_{2}\right)} \sigma, \sigma\right)=\left(e^{-\mu\left(0, R_{2}\right)},-e^{-\lambda\left(0, R_{2}\right)}\right)
$$

which means that we should choose $\sigma:=-e^{-\lambda\left(0, R_{2}\right)}$. Let $\tau_{1}$ and $\tau_{2}$ be the values of the affine parameter such that $(t, r)\left(\tau_{1}\right)$ is the intersection point of $\gamma_{2}$ with $\gamma_{1}$ and $(t, r)\left(\tau_{2}\right)$ is the intersection point of $\gamma_{2}$ with the line $r=R_{1}$, i.e., $r\left(\tau_{1}\right)=R^{*}$ and $r\left(\tau_{2}\right)=R_{1}$. For the affine length $L$ of the corresponding piece of $\gamma_{2}$ we find that

$$
\begin{aligned}
L & =\int_{\tau_{1}}^{\tau_{2}} d \tau=\int_{R^{*}}^{R_{1}} \frac{d r}{\sigma}=e^{\lambda\left(0, R_{2}\right)}\left(R^{*}-R_{1}\right) \\
& \geq\left(1-\frac{2 M}{R_{2}}\right)^{-1 / 2} \frac{R_{2}-R_{1}}{2}\left(1-\frac{2 M}{R_{1}}\right) \\
& \rightarrow \infty \text { as } R_{2} \rightarrow \infty .
\end{aligned}
$$

This proves the claim of Proposition 4.3 in the sense of [20].

We now turn to the question whether Theorem 4.1 also implies the formation of a black hole in some appropriate, coordinate-free sense. A maximal development of Cauchy data is said to contain a black hole if future null infinity $\mathcal{I}^{+}$is complete and the causal past $J^{-}\left(\mathcal{I}^{+}\right)$of future null infinity has a nonempty complement; cf. [23, Sect. 12]. Intuitively this says that no causal curve, i.e., no particle trajectory or light ray, originating in the complement of $J^{-}\left(\mathcal{I}^{+}\right)$can reach future null infinity, and that such trapped causal curves really exist. In the spacetimes obtained in Theorem 4.1 the null geodesic $\gamma^{*}$ does not reach future null infinity, and since we have shown that the latter is actually complete, $\gamma^{*}$ cannot reach future null infinity in any extension (such as the maximal development) either. Since $\mathcal{I}^{+}$is complete for the spacetimes we obtained, $\gamma^{*}$, in order to reach $\mathcal{I}^{+}$ in the maximal development, would have to enter the outer region $D ; \gamma^{*}\left(\tau^{*}\right) \in D$. But following $\gamma^{*}$ backwards we see that it must have stayed in $D$ for $\tau \leq \tau^{*}$ and hence cannot have reached the region $\{r>2 M\}$ to begin with.

In other words, we have in Theorem 4.1 constructed a globally hyperbolic spacetime $\mathcal{M}$ which has a complete $\mathcal{I}^{+}$and in which the complement of $J^{-}\left(\mathcal{I}^{+}\right)$is nonempty. If we consider the maximal development $\overline{\mathcal{M}}$ of the same Cauchy data, it has the same, complete $\mathcal{I}^{+}$, and since $\mathcal{M} \subset \overline{\mathcal{M}}$, the complement of $J^{-}\left(\mathcal{I}^{+}\right)$in $\overline{\mathcal{M}}$ is nonempty as well. Hence the following is true. 
Proposition 4.4. Initial data as specified in Theorem 4.1 lead to the formation of a black hole in the following sense. The spacetimes $\mathcal{M}$ obtained in Theorem 4.1 possess a complete future null infinity $\mathcal{I}^{+}$, and its causal past $J^{-}\left(\mathcal{I}^{+}\right)$has nonempty complement in the maximal development of the data.

5. Concluding remarks. In this final section we want to discuss the question to which extent the results from the previous section really depend on the particular matter model which we employed, we want to compare these results to previous results for other matter models, and we want to discuss future perspectives.

5.1. General matter models. The issue of gravitational collapse and in particular the validity of the cosmic censorship conjecture should of course be addressed not just for one particular matter model such as the collisionless gas. Indeed, some key arguments in the proof of Theorem 4.1 do depend only on certain general properties of the matter model and not on its specific nature. We briefly discuss this issue. To this end, let

$$
\rho:=e^{-2 \mu} T_{00}, p:=e^{-2 \lambda} T_{11}, j:=-e^{-\mu-\lambda} T_{01} .
$$

Firstly, we assume that the following two conditions are satisfied.

- The dominant energy condition holds.

- The radial pressure $p$ is nonnegative.

In general relativity the dominant energy condition (DEC) is the main criterion which a matter model must satisfy in order to be considered realistic; cf. [29]. The nonnegative pressure condition (NNP) is a standard assumption for most astrophysical models. These two criteria imply that

$$
0 \leq p \leq \rho \text { and }|j| \leq \rho
$$

cf. 29] and [35]. Furthermore, by (DEC), any world line $(s, R(s))$ of a material particle or a photon satisfies the estimate

$$
\left|\frac{d R}{d s}(s)\right| \leq e^{(\mu-\lambda)(s, R(s))}
$$

so that locally the speed of energy flow is less than or equal to the speed of light. In addition we need to assume certain a priori information on the behavior of the solutions of the Einstein-matter equations, namely, that for solutions launched by data from a suitable class,

- $\gamma^{+}$defined by (4.2) exists on $[0, \infty[$, and the solution exists on $D$,

- there exists a constant $c_{1}>0$ such that $\rho \leq-c_{1} j$ in $D$.

The role of the "global existence condition" (GLO) is obvious. The "gravitational collapse condition" (GCC) is crucial for our method of proof; in particular, together with (5.2), it implies that $j \leq 0$ in $D$, i.e., that the matter is ingoing for all times. Notice also that in (4.8), condition (GCC) was used. We emphasize that for Vlasov matter the conditions (DEC) and (NNP) hold always, while (GLO) and (GCC) follow via (4.7) from a suitable restriction of the initial data.

For a general matter model satisfying these assumptions, not all the results in Theorem 4.1 can be obtained, but the following still holds:

$$
\lim _{s \rightarrow \infty} \gamma^{+}(s)<\infty \text { and } \lim _{t \rightarrow \infty} \mu(t, r)=-\infty \text { for } \lim _{s \rightarrow \infty} \gamma^{+}(s) \leq r \leq r_{1}
$$


for some $r_{1}>\lim _{s \rightarrow \infty} \gamma^{+}(s)$. If $r^{*}$ and $\gamma^{*}$ are defined as in Theorem 4.1, then

$$
\lim _{s \rightarrow \infty} \gamma^{*}(s)<\infty
$$

and every radially outgoing null geodesic $\gamma$ with $\gamma(0)>r^{*}$ is future complete with $\lim _{s \rightarrow \infty} \gamma(s)=\infty$. The analysis in Section 4.3 applies to the resulting spacetimes as well, in particular since they again are vacuum for $r \geq R_{1}$.

5.2. Related results. Due to the inherent difficulties of the Einstein field equations, progress towards understanding the issues of cosmic censorship and the formation of black holes has up to now been restricted to the case of spherical symmetry. At least this assumption is made in all the papers we mention below.

The most complete understanding of the issues at hand has so far been obtained for the case where the Einstein equations are coupled to a massless scalar field; cf. [14, 15, 16, 17, 18, 19, 20. The final result of these investigations is that weak and strong cosmic censorship hold for the Einstein-scalar field system. A crucial step was the investigation in [16] where explicit conditions on the initial data were formulated which guarantee the formation of a trapped surface. Although the conditions we impose for Theorem 4.1 are reminiscent of the ones in [16, there is also an important difference. In [16] the admissible data cover the full range of $2 m / r \in] 0,1$ [, whereas for our result we need that initially $2 \mathrm{~m} / \mathrm{r}$ is close to one in the outer matter ring.

However, there may be a better reason for this difference than just the limited abilities of the present authors. The spherically symmetric Einstein-Vlasov system can exhibit at least the following qualitatively different types of behavior. Firstly, for small data the solution disperses in the sense of Theorem 3.2. Secondly, the system has a tremendously rich family of steady states; cf. [36, 41, 42] and also [8] concerning the possible shapes these steady states can take. Numerical investigations show clearly that there are both stable and unstable steady states; cf. [7. There is also strong numerical evidence [7] that the system has time-periodic or at least almost-periodic solutions, which is the third type of solution behavior. The fourth and last type of solution behavior is gravitational collapse and formation of a black hole as shown by Theorem 4.1. To our knowledge only dispersion and gravitational collapse are known as possible solution behaviors for the Einstein-scalar field system, and this wider range of qualitative solution behaviors for the Vlasov matter model may explain why the condition needed to force gravitational collapse is more restrictive than for the scalar field.

In passing we also note that the primary motivation for coupling the Einstein equations to a scalar field is, according to [20], to capture the wave nature of nonsymmetric vacuum solutions to the Einstein equations in a spherically symmetric setup while still enjoying the simplifications which the latter symmetry assumption entails. As mentioned above, the Vlasov matter model is actually used in astrophysics to describe galaxies or globular clusters.

Another matter model which features prominently in the history of the concepts of gravitational collapse and black holes is a fluid with pressure zero which is usually termed dust. The analysis in 32 has definitely shaped the overall picture of gravitational collapse to a large extent. The physical reason for neglecting pressure is the intuition that once the collapse is sufficiently advanced, gravity will dominate all other forces, including 
pressure. One important mathematical advantage of this matter model is that one can use coordinates which are co-moving with the matter. On the other hand, dust produces shell-crossing singularities which hinder the analysis of the real issues, and dust can lead to naked singularities as shown in [13. Whether cosmic censorship will eventually be established for Vlasov matter or not, solutions of the Einstein-Vlasov system do have nontrivial pressure, and the system has so far not been shown to produce naked singularities.

In making the last statement we are fully aware of [50. There it is claimed that numerical simulations of the axially symmetric Einstein-Vlasov system can lead to naked singularities. However, there is reason to believe that the matter model which was actually simulated in [50] was dust and not Vlasov; cf. [45].

To conclude this comparison with previously known results, we mention that in 45 a continuity argument was used to show that there exist initial data for the spherically symmetric Einstein-Vlasov system which lead to the formation of trapped surfaces. Combining this with the results of [21] and [23] implies that there exist data which lead to the formation of a black hole. Due to the method of proof in [45], this analysis does not give explicit conditions on the data which guarantee this type of behavior. Our approach does produce such explicit conditions on the data, and these data are stable against perturbations which are small in a suitable sense.

5.3. Open problems and future perspectives. To conclude we mention some open problems and possible perspectives for future research. An immediate question is whether the geodesic $\gamma^{*}$ in Theorem 4.1 is future complete; the question whether an event horizon has complete generators is of general interest. This issue is closely related to the question whether in the limit $t \rightarrow \infty$ all the matter ends up in the region $\{r \leq 2 M\}$, i.e., whether $\lim _{t \rightarrow \infty} m(t, 2 M)=2 M$. It is clear from the geodesic equation for $\gamma^{*}$ that this geodesic cannot be complete if it is running in vacuum all the time, cf. (4.10), which means that at least some matter must cross $r=2 M$ if $\gamma^{*}$ should be complete. These questions are currently under investigation by two of the authors, and we believe that for suitably restricted data indeed $\lim _{t \rightarrow \infty} m(t, 2 M)=2 M$.

The next logical step will be to analyze the situation corresponding to Theorem 4.1 in a coordinate system which has the potential to cover regions of spacetime containing trapped surfaces and which may reach all the way to the spacetime singularity which forms at the center. Preliminary steps in this direction look promising.

Much more demanding is the question of how to relax the conditions in Theorem 4.1 so that the numerical observations reported in [7] where the perturbation of unstable steady states leads to the formation of black holes are covered. An analytic understanding of the stability properties, which were observed numerically, is a further open problem. An answer to this could also help to explain the phenomenon of critical collapse; cf. [7, 31, 44, for relevant numerical results for the Einstein-Vlasov system and 28, for a general discussion of this issue.

As pointed out above, the question whether weak cosmic censorship holds for the spherically symmetric Einstein-Vlasov system is related to the question whether this system has global solutions in Schwarzschild coordinates or not. Useful estimates in the direction of global existence in Schwarzschild coordinates, which go beyond what was reported in Section 3, were established in 2, but the problem remains open. When 
thinking about weak cosmic censorship one should definitely keep the possibility in mind that global existence in Schwarzschild coordinates could be violated for an initial data set "of measure zero", while weak cosmic censorship could still be true for the spherically symmetric Einstein-Vlasov system.

Eventually one will wish to go beyond spherical symmetry, and any extension of the results mentioned in these notes to, for example, the case of axial symmetry is in our opinion a challenging and worthwhile problem.

Acknowledgement. The authors would like to thank Piotr Chruściel, Helmut Friedrich, and Alan Rendall for valuable discussions.

\section{REFERENCES}

[1] H. Andréasson, The Einstein-Vlasov System/Kinetic Theory, Living Rev. Relativity 5 (2002). MR $1966539(2004 \mathrm{c}: 83009)$

[2] H. Andréasson, On global existence for the spherically symmetric Einstein-Vlasov system in Schwarzschild coordinates, Indiana Univ. Math. J. 56, 523-552 (2007). MR2317537(2008d:83016)

[3] H. Andréasson, On static shells and the Buchdahl inequality for the spherically symmetric EinsteinVlasov system, Commun. Math. Phys. 274, 409-425 (2007) MR2322910 (2008h:83048)

[4] H. Andréasson, Sharp bounds on $2 m / r$ of general spherically symmetric static objects, J. Differential Equations 245, 2243-2266 (2008). MR2446191

[5] H. Andréasson, M. Kunze, G. Rein, Global existence for the spherically symmetric Einstein-Vlasov system with outgoing matter, Comm. Partial Differential Eqns. 33, 656-668 (2008). MR.2424372

[6] H. Andréasson, M. Kunze, G. Rein, The formation of black holes in spherically symmetric gravitational collapse, preprint 2008, arXiv: gr-qc/0706.3787. MR2424372

[7] H. Andréasson, G. Rein, A numerical investigation of the stability of steady states and critical phenomena for the spherically symmetric Einstein-Vlasov system, Classical Quantum Gravity 23, 3659-3677 (2006). MR2235427(2007d:83073)

[8] H. Andréasson, G. Rein, On the steady states of the spherically symmetric Einstein-Vlasov system, Classical Quantum Gravity 24, 1809-1832 (2007). MR2310358(2008b:83054)

[9] C. Bardos, P. Degond, Global existence for the Vlasov-Poisson system in 3 space variables with small initial data, Ann. Inst. Henri Poincaré, Analyse non linéaire 2, 101-118 (1985). MR794002 (86k:35129)

[10] J. Batt, Global symmetric solutions of the initial value problem in stellar dynamics, J. Diff. Eqns. 25, 342-364 (1977). MR0487082 (58:6749)

[11] J. Binney, S. Tremaine, Galactic Dynamics, Princeton University Press, 1987.

[12] Y. Choquet-Bruhat, Problème de Cauchy pour le système intégro différentiel d'Einstein-Liouville, Ann. Inst. Fourier 21, 181-201 (1971). MR0337248 (49:2018)

[13] D. Christodoulou, Violation of cosmic censorship in the gravitational collapse of a dust cloud, Comm. Math. Phys. 93, 171-195 (1984). MR742192 (86a:83058)

[14] D. Christodoulou, The problem of a self-gravitating scalar field, Comm. Math. Phys. 105, 337-361 (1986). MR848643 (87i:83009)

[15] D. Christodoulou, A mathematical theory of gravitational collapse, Comm. Math. Phys. 109, 613647 (1987). MR885564 (88f:83005b)

[16] D. Christodoulou, The formation of black holes and singularities in spherically symmetric gravitational collapse, Comm. Pure Appl. Math. 44, 339-373 (1991). MR.1090436(92d:83044)

[17] D. Christodoulou, Bounded variation solutions of the spherically symmetric Einstein-scalar field equations, Comm. Pure Appl. Math. 46, 1131-1220 (1993). MR.1225895 (95b:35221)

[18] D. Christodoulou, Examples of naked singularity formation in the gravitational collapse of a scalar field, Ann. of Math. (2) 140, 607-653 (1994). MR1307898(95j:83100)

[19] D. Christodoulou, The instability of naked singularities in the gravitational collapse of a scalar field, Ann. of Math. (2) 149, 183-217 (1999). MR1680551(2000a:83086)

[20] D. Christodoulou, On the global initial value problem and the issue of singularities, Classical Quantum Gravity 16, A23-A35 (1999). MR.1728432 (2001a:83010) 
[21] M. Dafermos, Spherically symmetric spacetimes with a trapped surface, Classical Quantum Gravity 22, 2221-2232 (2005). MR2145241 (2006a:83034)

[22] M. Dafermos, A note on the collapse of small data self-gravitating massless collisionless matter, $J$. Hyperbolic Differential Equations 3, 589-598 (2006). MR2289606 (2008f:83019)

[23] M. Dafermos, A. D. Rendall, An extension principle for the Einstein-Vlasov system in spherical symmetry, Ann. Henri Poincaré 6, 1137-1155 (2005). MR2189379(2007c:83011)

[24] A. Einstein, Zur Allgemeinen Relativitätstheorie, Sitzungsber. der Preuss. Akad. der Wissenschaften zu Berlin; 1915, 778-786 (1915).

[25] A. Einstein, Die Feldgleichungen der Gravitation, Sitzungsber. der Preuss. Akad. der Wissenschaften zu Berlin; 1915, 844-847 (1915).

[26] R. T. Glassey, W. A. Strauss, Singularity formation in a collisionless plasma could occur only at high velocities, Arch. Rat. Mech. Anal. 92, 59-90 (1986). MR816621 (87j:82064)

[27] R. T. Glassey, W. A. Strauss, Absence of shocks in an initially dilute collisionless plasma, Commun. Math. Phys. 113, 191-208 (1987). MR919231 (88k:76034)

[28] C. Gundlach, Critical phenomena in gravitational collapse, Living Rev. Relativity 2 (1999). MR 1728882(2001a:83061)

[29] S. Hawking, G. F. R. Ellis, The Large Scale Structure of Space-time, Cambridge University Press 1975. MR0424186 (54:12154)

[30] P.-L. Lions, B. Perthame, Propagation of moments and regularity for the 3-dimensional VlasovPoisson system, Invent. Math. 105, 415-430 (1991). MR.1115549(92e:35160)

[31] I. Olabarrieta, M. W. Choptuik, Critical phenomena at the threshold of black hole formation for collisionless matter in spherical symmetry, Phys. Rev. D. 65, 024007 (2002). MR.1892138(2003a:83048)

[32] J. R. Oppenheimer, H. Snyder, On continued gravitational contraction, Phys. Rev. 56, 455-459 (1939).

[33] R. Penrose, Gravitational collapse and space-time singularities, Phys. Rev. Lett. 14, 57-59 (1965). MR0172678 (30:2897)

[34] K. Pfaffelmoser, Global classical solutions of the Vlasov-Poisson system in three dimensions for general initial data, J. Differential Equations 95, 281-303 (1992). MR1165424 (93d:35170)

[35] E. Poisson, A Relativist's Toolkit. The Mathematics of Black-hole Mechanics, Cambridge University Press, 2004. MR2063287 (2005d:83002)

[36] G. Rein, Static solutions of the spherically symmetric Vlasov-Einstein system. Math. Proc. Camb. Phil. Soc. 115, 559-570 (1994). MR1269939 (95b:83054)

[37] G. Rein, The Vlasov-Einstein System with Surface Symmetry, Habilitationsschrift, München, 1995.

[38] G. Rein, Collisionless Kinetic Equations from Astrophysics-The Vlasov-Poisson System, Handbook of Differential Equations, Evolutionary Equations. Vol. 3. Eds. C. M. Dafermos and E. Feireisl, Elsevier (2007).

[39] G. Rein, A. D. Rendall, Global existence of solutions of the spherically symmetric Vlasov-Einstein system with small initial data, Comm. Math. Phys. 150, 561-583 (1992). Erratum: Comm. Math. Phys. 176, 475-478 (1996). MR.1204320 (94c:83028)

[40] G. Rein, A. D. Rendall, The Newtonian limit of the spherically symmetric Vlasov-Einstein system, Comm. Math. Phys. 150, 585-591 (1992). MR1204321 (94c:83029)

[41] G. Rein, A. D. Rendall, Smooth static solutions of the spherically symmetric Vlasov-Einstein system. Ann. de l'Inst. H. Poincaré, Physique Théorique 59, 383-397 (1993). MR.1254978 (94m:35296)

[42] G. Rein, A. D. Rendall, Compact support of spherically symmetric equilibria in non-relativistic and relativistic galactic dynamics. Math. Proc. Camb. Phil. Soc. 128, 363-380 (2000). MR.1735298 $(2001 \mathrm{k}: 85001)$

[43] G. Rein, A. D. Rendall, J. Schaeffer, A regularity theorem for solutions of the spherically symmetric Vlasov-Einstein system, Comm. Math. Phys. 168, 467-478 (1995). MR1328249 (96g:58187)

[44] G. Rein, A. D. Rendall, J. Schaeffer, Critical collapse of collisionless matter - a numerical investigation, Phys. Rev. D 58, 044007 (1998).

[45] A. D. Rendall, Cosmic censorship and the Vlasov equation, Classical Quantum Gravity 9, L99-L104 (1992). MR1178792 (93e:83052)

[46] A. D. Rendall, The Newtonian limit for asymptotically flat solutions of the Vlasov-Einstein system. Comm. Math. Phys. 163, 89-112 (1994). MR1277935 (95e:83026)

[47] A. D. Rendall, An introduction to the Einstein-Vlasov system, Banach Center Publications 41, 35-68 (1997). MR 1466508 (98k:83007) 
[48] A. D. Rendall, Theorems on existence and global dynamics for the Einstein equations, Living Rev. Relativity 6 (2005). MR.1932418(2003h:83016)

[49] K. Schwarzschild, Über das Gravitationsfeld eines Massenpunktes nach der Einstein'schen Theorie, Sitzungsber. der Preuss. Akad. der Wissenschaften zu Berlin; 1916, 189-196 (1916).

[50] S. Shapiro, S. Teukolsky, Formation of naked singularities: The violation of cosmic censorship, Phys. Rev. Letters 66, 994-997 (1991). MR1095978 (91k:83058)

[51] R. Wald, General Relativity, Chicago University Press, 1984. MR757180 (86a:83001) 